\title{
Molecular Characterization of Leading Edge Protrusions in the Absence of Arp2/3 Complex.
}

\author{
Karen L Anderson ${ }^{1}$, Christopher Page ${ }^{1}$, Praveen Suraneni ${ }^{2}$, Rong Li $^{2}$, Niels Volkmann ${ }^{1}$ and Dorit \\ Hanein $^{1}$ \\ ${ }^{1}$ Bioinformatics and Systems Biology Program, Sanford-Burnham Medical \\ Research Institute, La Jolla, CA 92037 \\ ${ }^{2}$ Stowers Institute for Medical Research, Kansas City, MO 64110
}

Cells employ protrusive leading edges to navigate and promote their migration in diverse physiological environments. Classical models of leading edge protrusion rely on a treadmilling dendritic actin network that undergoes continuous assembly nucleated by the Arp $2 / 3$ complex, forming ruffling lamellipodia. Although the dendritic nucleation model has been rigorously evaluated in several computational studies, experimental evidence demonstrating a critical role for Arp $2 / 3$ in the generation of protrusive actin structures and cell motility has been far from clear. Most components of the pathway have been probed for their relevance by RNA interference or dominant-negative constructs. However, given that the Arp2/3 complex nucleates actin at nanomolar concentrations, even a dramatic knockdown could still leave behind a level sufficient to fully or partially support Arp2/3 complex-dependent functions.

Our recent work renders the characterization of newly developed fibroblasts cells completely lacking the Arp2/3 complex. Characterization of the impact of the absence of Arp $2 / 3$ complex on these genetically matched cells with and without Arp2/3 complex included single cell spreading assays, wound healing assays, long-time single cell motility tracking, chemotaxis assays, fluorescence staining imaging with confocal or structured illumination microscopy [1.2]. In the absence of Arp2/3 complex, the fibroblasts were unable to extend lamellipodia but generated dynamic leading edges composed primarily of filopodia-like protrusions (FLPs), with formin proteins (mDia1 and mDia2) concentrated near their tips. ARPC3-/- fibroblasts maintained an ability to move but exhibited a strong defect in persistent directional migration in both wound healing and chemotaxis assays $[1,2]$.

In the absence of the Arp2/3 complex in ARPC3-/- fibroblasts, formins are required for the extension of FLPs but they are insufficient to produce a continuous leading edge. We hypothesize that the leading edge protrusions of these motile cells are driven by local coordinated actions of neighboring FLPs and myosin-II-mediated contractility localized in the arcs in between the two respective FLPs. Our cryoEM studies provided direct evidence for the existence of short isotropic actin filaments in the arcs regions. Here, we will highlight our advances on determining the molecular-level organization of these actin networks, through an integrated approach that employs electron cryo-tomography of whole mammalian cells in conjunction with correlative light microscopy.

This work is supported by NIGMS grant P01 GM066311. 
References:

[1] Suraneni P, Rubinstein B, Unruh JR, Durnin M, Hanein, D, and Li R (2012). The Arp2/3 complex is required for lamellipodia extension and directional fibroblast cell migration. J Cell Biol. 197, 239. PMC 3328382

[2] Suraneni P, Fogelson B, Rubinstein B, Noguera P, Volkmann N, Hanein D, Mogilner A, Li R (2015). A mechanism of leading edge protrusion in the absence of Arp2/3 complex. Mol Biol Cell. Jan 7 [Epub ahead of print].

A

$\mathrm{ARPC}^{+/+}$

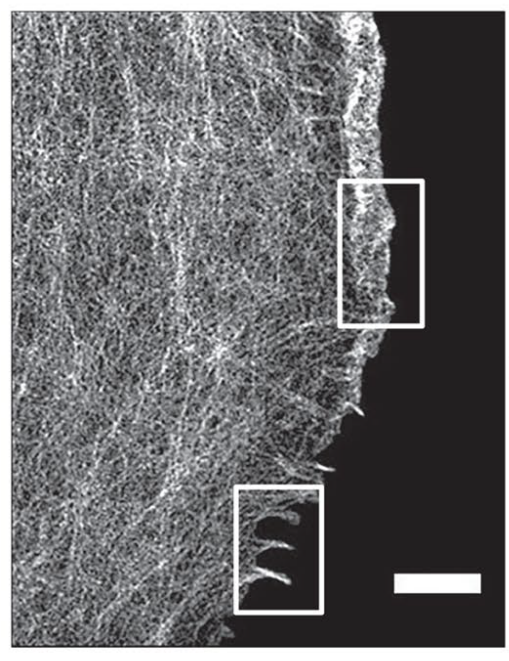

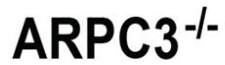

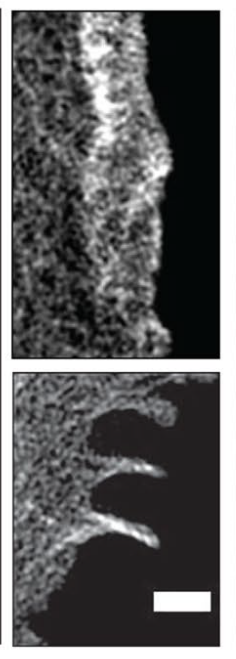

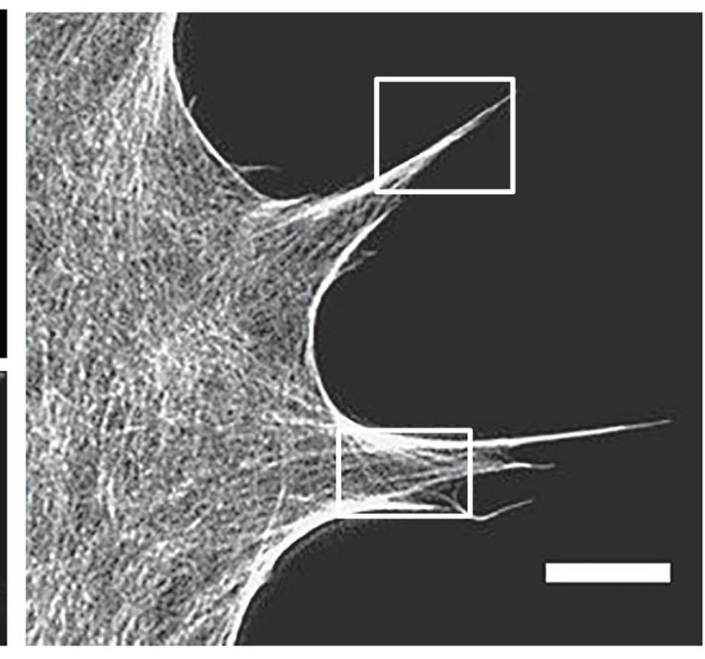

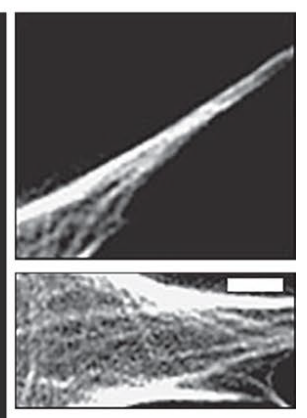

Figure 1: Actin organization at the leading edge in wt and ARPC3-/- fibroblasts. (A) wt and ARPC3-/cells were stained with fluorescent phalloidin and imaged by SIM [2]. The white boxes represent the zoomed regions in the corresponding images. Scale bars: (A) wt $2 \mu \mathrm{m}, 1.5 \mu \mathrm{m}$ (zoom) and ARPC3-/$2.5 \mu \mathrm{m}, 1.5 \mu \mathrm{m}$. The corresponding molecular actin organization of these cells will be the center of this study. 